\title{
Aldehyde dehydrogenase 1 expression has prognostic significance in patients with glioma
}

\author{
JUN WANG $^{1}$, CHENG-LIANG YANG $^{1}$ and LI-LI ZOU ${ }^{1,2}$ \\ ${ }^{1}$ Translational Neuroscience and Neural Regeneration and Repair Institute/Institute of Cell Therapy, \\ The First Hospital of Yichang; ${ }^{2}$ Department of Microbiology and Immunology, Medical College, \\ China Three Gorges University, Yichang, Hubei 443002, P.R. China
}

Received August 25, 2016; Accepted May 19, 2017

DOI: $10.3892 /$ mco.2017.1396

\begin{abstract}
As a cancer stem cell marker associated with tumorigenesis, aldehyde dehydrogenase 1 (ALDH1) has recently been identified in gliomas. However, an insufficient number of clinical studies have been published to demonstrate its prognostic significance in glioma. In the present study, a systematic meta-analysis was performed to comprehensively evaluate the correlation of ALDH1 with age, sex, the World Health Organization (WHO) grade, and overall survival (OS) in patients with glioma. A search of relevant publications was conducted to select eligible studies on this subject, and the pooled hazard ratios (HRs) and related risks (RRs) with $95 \%$ confidence intervals (95\% CIs) were assessed. Publication bias was also evaluated using Begg's funnel plots. A total of 6 articles were identified that included a total of 1,057 patients. OS analysis revealed that a high expression of ALDH1 was significantly associated with poor 5-year OS (n=6; HR, 2.10; 95\% CI, 1.13-3.91; $\mathrm{P}<0.0001$ ), and a high WHO grade (III+IV; $\mathrm{n}=4$; RR, 2.28; 95\% CI, 1.31-3.99; $\mathrm{P}=0.001)$. In conclusion, a high expression of ALDH1 is associated with a high WHO grade of gliomas and a worse prognosis in patients with glioma. Further, well-designed clinical studies are required to confirm its role in the process of selecting a suitable therapeutic approach in glioma.
\end{abstract}

\section{Introduction}

As a heterogeneous disease, glioma is one of the most common types of human brain cancer, exhibiting malignant aggressiveness and poor survival (1). Despite multimodal aggressive treatment, comprising surgery and radiation with chemotherapeutics, there has been no marked improvement in the median

Correspondence to: Dr Li-Li Zou, Department of Microbiology and Immunology, Medical College, China Three Gorges University, 8 Daxue Road, Xiling, Yichang, Hubei 443002, P.R. China

E-mail: zoulili@mail3.sysu.edu.cn

Key words: aldehyde dehydrogenase 1, World Health Organization grade, gliomas, prognosis, meta-analysis survival time of patients with World Health Organization (WHO) high-grade (III+IV) glioma following diagnosis, which mainly ranges from 12 to 5 months (2). Histopathologically, glioma is a heterogeneous disease, divided into four grades and different types according to WHO, i.e., astrocytomas, glioblastoma multiforme (GBM), medulloblastoma, and ependymocytoma (3). As a life-threatening disease, the underlying molecular mechanism of glioma has yet to be comprehensively elucidated, and merits further investigation.

The recent discovery of a small subpopulation of cancer cells termed 'cancer stem cells' (CSCs) has provided a novel conceptual paradigm of how cancers initialize, progress, and metastasize due to their abilities of chemoresistance, DNA repair, resistance to enter apoptosis, and formation of the tumor bulk $(4,5)$. In brain tumors, different types of stem cell markers may be used to identify CSCs, including CD133, Sox2, and CD44 (6,7), which are positively associated with pathological grade, and negatively associated with overall survival (OS) and progression-free survival (PFS) in patients with glioma $(8,9)$.

Aldehyde dehydrogenase $(\mathrm{ALDH})$ is a large enzyme superfamily that comprises 19 different members (10). As a detoxifying enzyme, ALDH1 is able to oxidize retinol to retinoic acid in the early stages of stem cell differentiation, and it has therefore been claimed to be a novel hallmark of CSCs in a variety of malignancies $(11,12)$. High levels of ALDH1 expression are positively correlated with clonogenic capacity in GBM (13), medulloblastoma (14), and glioblastoma (15) cell lines. However, the number of clinical studies remains insufficient to enable its prognostic significance in glioma to be determined.

In the present study, a systematic meta-analysis of published eligible studies was performed to assess the correlation of ALDH1 expression with its prognostic significance in patients with glioma. This analysis may aid the identification of a novel biomarker that could enable the development of a novel therapeutic strategy for treating this challenging disease.

\section{Data collection methods}

Publication search. An assessment of the relevant publications was performed without any limitations of origin or language in the following databases: PubMed, Web of Science, 
MEDLINE, Google Scholar, Wanfang, Embase and CNKI, for publications up to October 2015. Various combinations of keywords as search terms were used, as follows: ('Aldehyde dehydrogenase 1' OR 'ALDH1') AND ('gliomas' OR 'glioma' OR 'astrocytomas' OR 'glioblastoma multiforme' OR 'GBM' OR 'medulloblastoma' OR 'ependymocytoma') AND ('expression' OR 'prognosis' OR 'prognostic' OR 'outcome' OR 'survival'). Potentially applicable reports, relevant reviews, and supplied additional data were investigated manually when essential data were not available from the original literature.

Eligibility criteria. The eligibility of all publications was assessed by two independent investigators (J.W. and L.-L.Z.), and discrepancies were resolved by discussion. Inclusion criteria were as follows: i) glioma patients without any limitation of types; ii) the outcomes of interest were age, sex, WHO grade, OS, progression free survival (PFS), and clinicopathological indicators of patients associated with ALDH1 expression; iii) the ALDH1 expression data could be used to determine the hazard ratios (HRs) or relative risks (RRs) with 95\% confidence intervals (95\% CIs); and iv) publications in Chinese and English. Exclusion criteria were as follows: i) abstracts, editorials, letters, expert opinions, or reviews; ii) non-human research; iii) the publication contained insufficient data; iv) studies with overlapping data; and v) the older of the publications from which the data were retrieved was excluded, in cases of data duplication.

Data extraction. To reduce the bias and enhance the credibility, relevant data extraction was performed by two independent observers (J.W. and L.-L.Z.) using standardized criteria as follows: First author, country of population, publication year, number of included patients (sample size), mean ages, histological type, study methods, WHO grade, cut-off values of ALDH1, and positive percentage.

Two independent observers (J.W. and L.-L.Z.) assessed the quality of all the included studies according to the four dimensions of methodology: i) the scientific design; ii) the laboratory methodology; iii) generalizability; and iv) the results analysis (16). The maximum score possible was 40 points (10 points for each dimension). The final scores show the percentage of the maximum achievable score (0-100\%).

Statistical analysis. STATA version 12.0 was the software predominantly used to perform the statistical analysis. Engauge Digitizer software (version 6.0) was used to extract data in the tables, text or/and figures from the included eligible papers. In addition, methods described by Tierney et al (17) and Parmar et al (18) were also used in the present analysis.

In order to evaluate the heterogeneity between different study results, the Chi-square and Cochran Q statistical tests were used. If the heterogeneity was determined to be statistically significant (defined as $\mathrm{P}<0.05$ ), a random-effects model would be performed; however, if no statistical heterogeneity existed (defined as $\mathrm{P}>0.1$ ), a fixed-effects model was used. In addition, the publication bias was assessed using Begg's funnel plots test (19), and sensitivity analysis was applied to assess the influence of a single study on the overall evaluation.

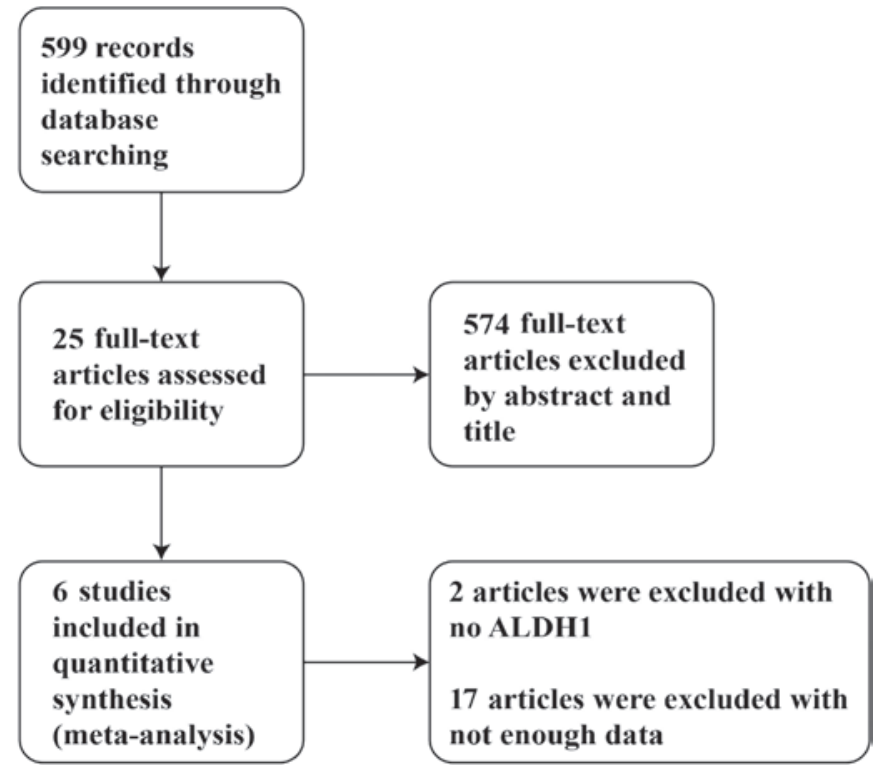

Figure 1. Literature search and selection of articles. Six articles were eventually included according to the inclusion criteria.

\section{Results}

Search results and characteristics of studies. The steps taken to search for relevant publications are described in detail in Fig. 1. A total of 599 publications were selected and identified according to the inclusion criteria described above. After an independent reading of the titles and abstracts by the two investigators (J.W. and L.-L.Z.), 574 articles were excluded due to being irrelevant to the subject, based on the exclusion criteria described above. The remaining 25 articles were further evaluated by reading the text thoroughly, and 6 studies remained, with 19 articles being excluded; 2 were not associated with ALDH1, and 17 lacked sufficient data.

The main characteristics of these 6 eligible studies are summarized in Table I (20-25). The publication years ranged from 2011 to 2015. Three studies were conducted in German populations, and three other studies were from China. The total number of patients with glioma was 1,057 , ranging from 70 to 298 patients per study. The percentage of positive ALDH1 expression varied from 21.5 to $90.9 \%$. Four articles were concerned with OS, 2 studies were on PFS, 1 study was concerned with median survival, and 1 article was about the HR. Two methods were essentially used to assess ALDH1 expression in patients with glioma, i.e. immunohistochemistry (IHC; 5 articles), and tissue microarray (TMA; 1 article).

Association between ALDH1 expression and OS of glioma. The time-to-event outcome is most appropriately analyzed using the HR, which takes into account the number and timing of events. There were 6 articles assessing the correlation of ALDH1 expression with OS in patients with glioma. Obvious heterogeneity was identified among those eligible studies, and the random-effects model was performed to evaluate the pooled HR. The results revealed that high ALDH1 expression was associated with poorer OS in patients with glioma (HR=2.10; 95\% CI, 1.13-3.91; P<0.0001) (Fig. 2). 
Table I. Main characteristics of the studies in the meta-analysis.

\begin{tabular}{llclcccccc}
\hline Authors, year & Country & $\begin{array}{c}\text { No. of } \\
\text { patients }\end{array}$ & Histology & $\begin{array}{c}\text { Mean } \\
\text { age }\end{array}$ & $\begin{array}{c}\text { No. of } \\
\text { males }\end{array}$ & $\begin{array}{c}\text { WHO } \\
\text { grade }\end{array}$ & Method & Cut-off & Positive \\
(Refs.)
\end{tabular}

WHO, World Health Organization; IHC, immunohistochemistry; TMA, tissue microassay.

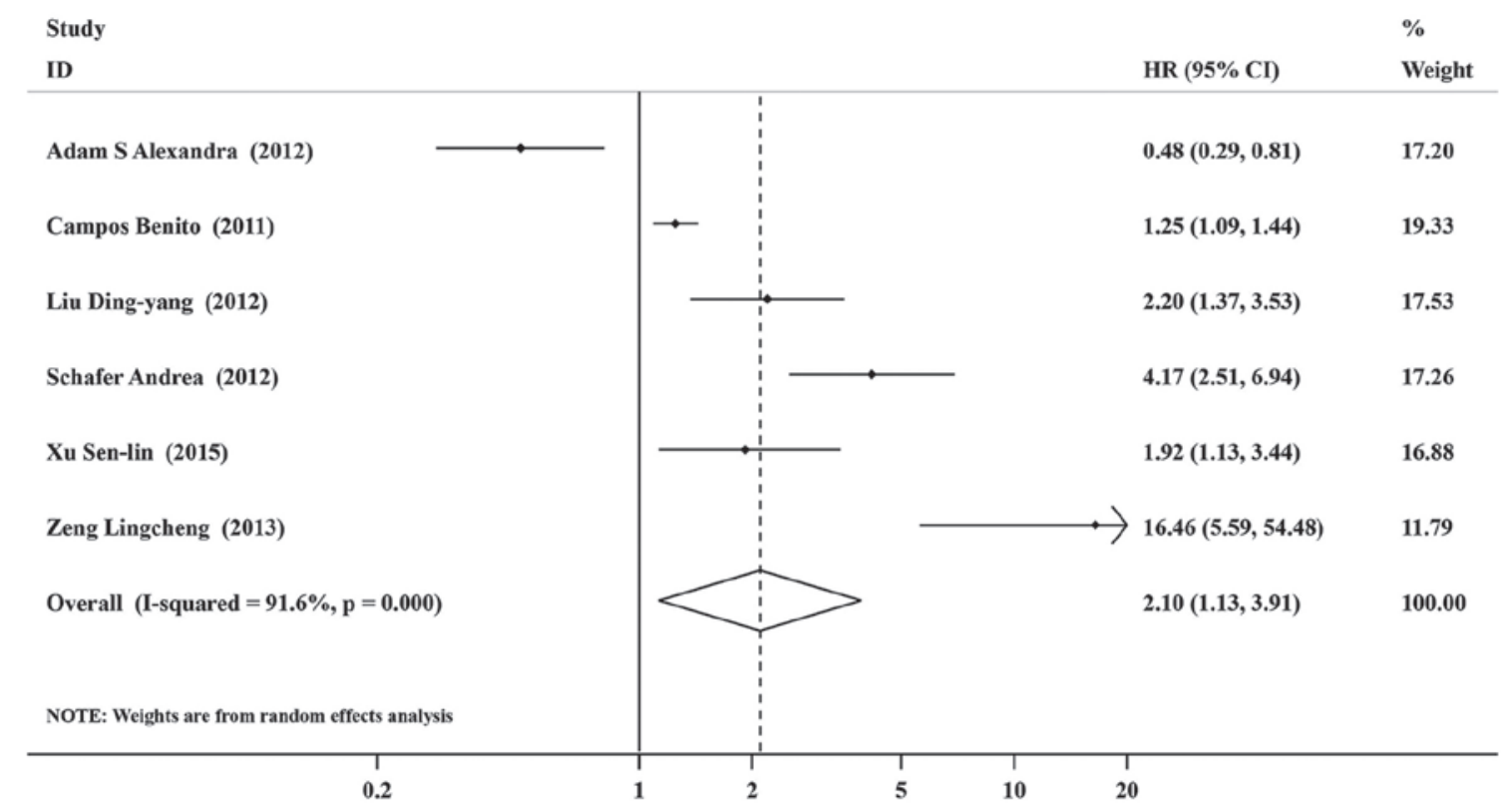

Figure 2. High aldehyde dehydrogenase 1 expression is associated with poorer overall survival in patients with glioma. HR, hazard ratio; CI, confidence interval.

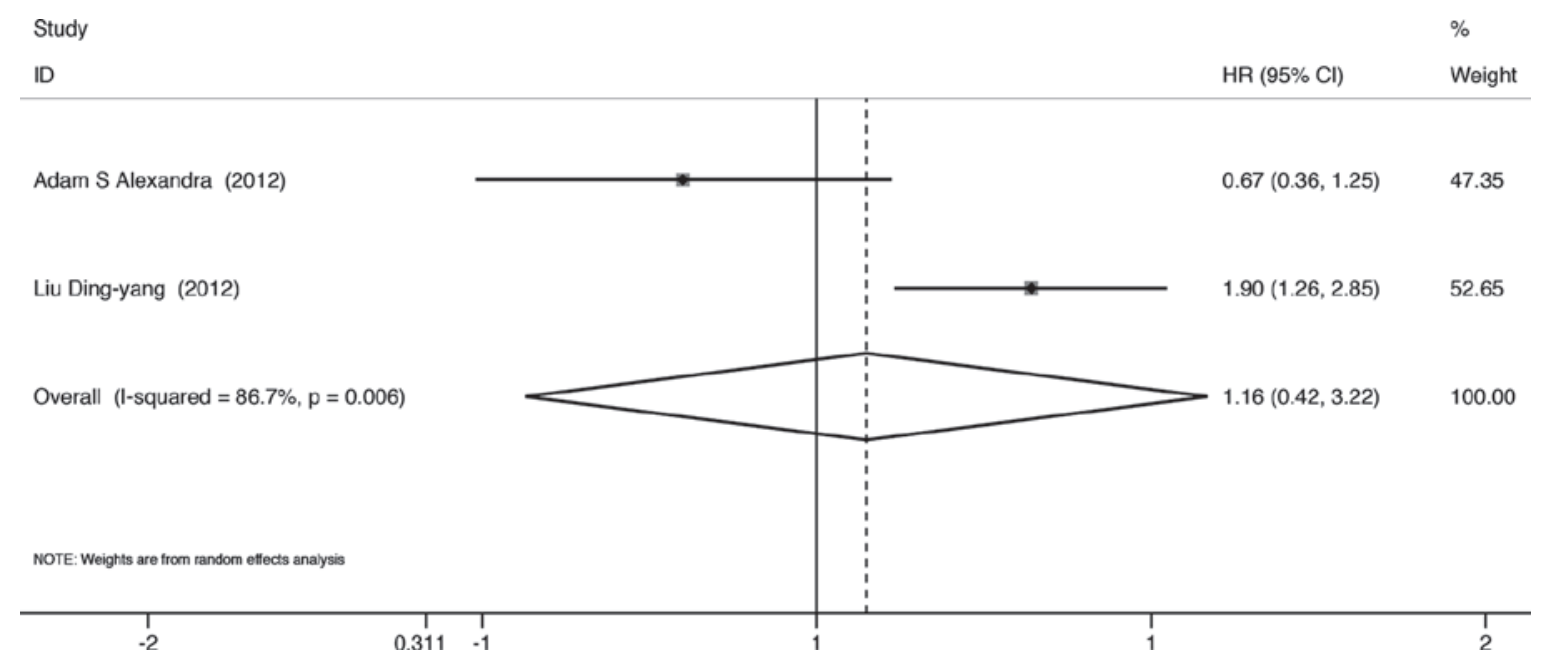

Figure 3. Association between aldehyde dehydrogenase 1 and the progression-free survival of patients with glioma. Individual and pooled HRs with $95 \%$ CIs are shown. HR, hazard ratio; CI, confidence interval.

To evaluate the association between ALDH1A1 and the PFS for the patients with glioma, survival analysis was performed.
As shown in Fig. 3, the meta-analysis results revealed that patients with glioma with positive ALDH1A1 expression had 


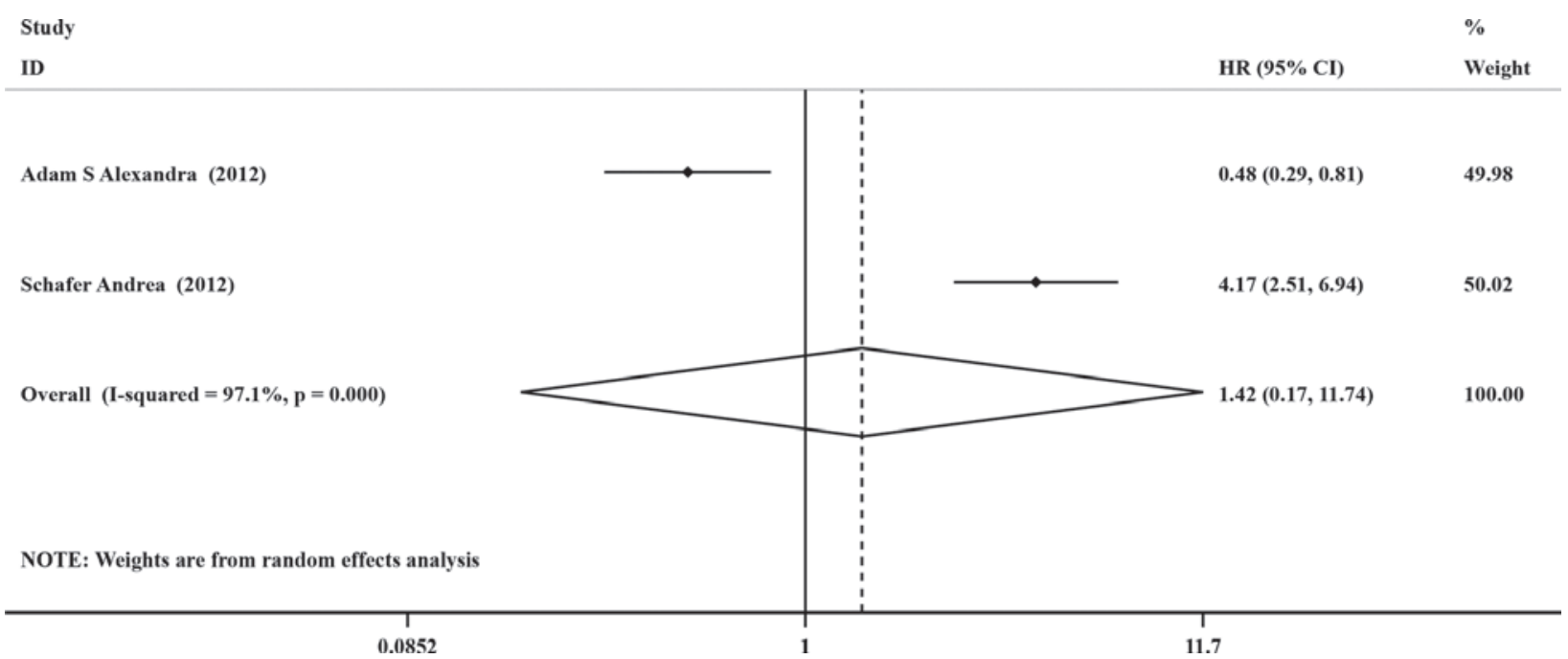

Figure 4. Association between aldehyde dehydrogenase 1 and the progression-free survival in patients with GBM. The individual and pooled HRs with 95\% CIs are shown. HR, hazard ratio; CI, confidence interval.

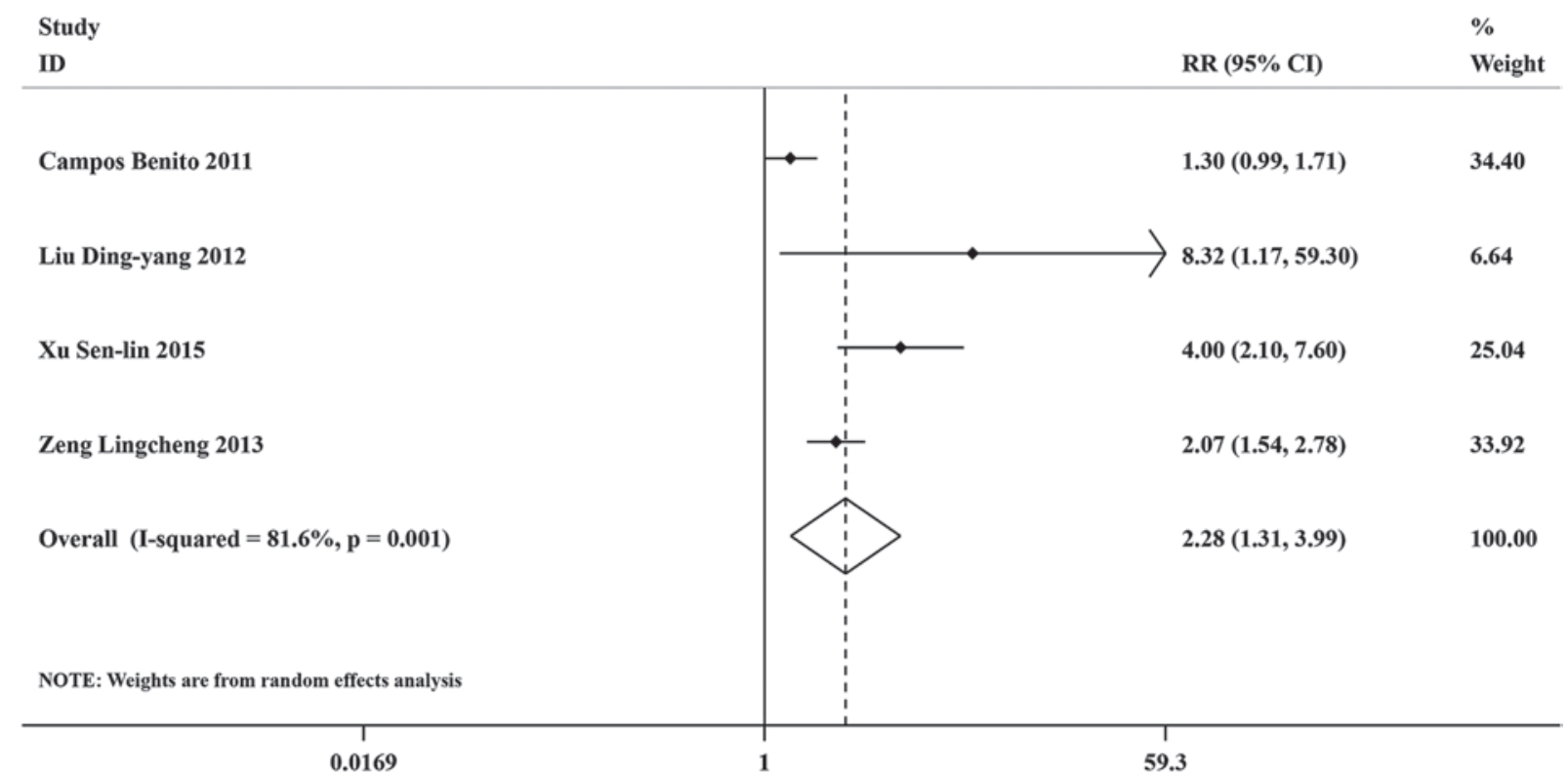

Figure 5. High aldehyde dehydrogenase 1 expression is associated with a high WHO grade in patients with glioma. RR, relative risk; CI, confidence interval; WHO, World Health Organization.

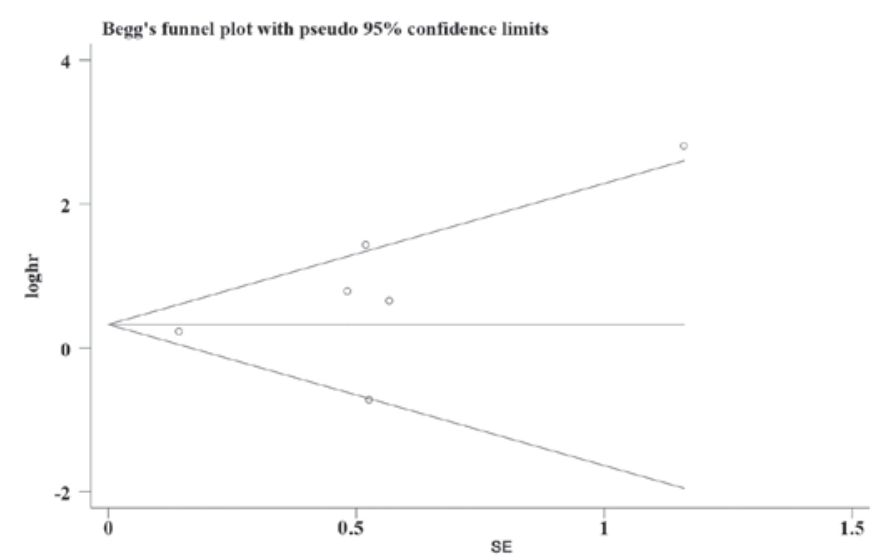

Figure 6. Funnel plot for the meta-analysis of the association between aldehyde dehydrogenase 1 expression and overall survival in patients with glioma. SE, standard error; loghr, logarithm of the hazard ratio. a worse PFS compared with those with negative expression $(\mathrm{HR}=1.16$; 95\% CI, 0.42-3.22; $\mathrm{P}=0.006$ ) (Fig. 3). To evaluate the association between ALDH1A1 and prognosis in patients with GBM, a subgroup analysis focusing solely on GBM and PFS was performed. As shown in Fig. 4, this meta-analysis revealed that patients with GBM with positive ALDH1A1 expression had a worse PFS compared with those with negative expression (HR=1.42; 95\% CI, 0.17-11.74; $\mathrm{P}<0.0001)$ (Fig. 4).

Correlation of ALDH1 expression with the WHO grade of glioma. RR, a parameter that measures only the number of events, is appropriate for measuring a dichotomous outcome. There were 4 articles assessing the correlation of ALDH1 expression with WHO grade in patients with glioma. Clear heterogeneity was identified comparing between the eligible studies, and the random-effects model was used to evaluate 
the pooled RR. The results demonstrated that high ALDH1 expression was associated with a high WHO grade (III + IV) in patients with glioma $(\mathrm{RR}=2.28 ; 95 \% \mathrm{CI}, 1.31-3.99 ; \mathrm{P}=0.001)$ (Fig. 5).

Assessment of publication bias. A Begg's funnel plot was used to assess publication bias of the present meta-analysis. The ALDH1 expression with OS was shown to be symmetric, and the P-value of Egger's test was 0.284>0.1 (Fig. 6), which indicated that there is no evident risk of publication bias in the meta-analysis on OS.

\section{Discussion}

Brain tumor growth is a CSC-dependent activity that has self-renewal capacity (26). In gliomas, CSCs are at least, in part, responsible for causing resistance to chemotherapeutic treatments and the poor prognosis of the disease (13). Therefore, identification of CSC markers is essential for the development of novel treatment strategies for gliomas.

Although ALDH1 has been identified as a novel CSC biomarker in gliomas (13), insufficient clinical data are available to enable the identification of its prognostic significance in patients with glioma. Therefore, a systematic meta-analysis was performed in the present study to evaluate the association between ALDH1 and age, sex, OS, and the WHO grade in patients with glioma. Six eligible studies were identified and included, and the pooled HRs and RRs with 95\% CIs were calculated.

In the present systematic meta-analysis, the outcome of 1,057 patients with glioma from 6 relevant articles associated with ALDH1, prognosis, and pathology were summarized. OS analysis demonstrated that there was an obvious correlation of a high expression of ALDH1 with poor 5-year OS $(n=6$; HR, 2.10; 95\% CI, 1.13-3.91; $\mathrm{P}<0.0001)$. In addition, high levels of ALDH1 expression in patients with glioma were positively associated with a high WHO grade (III+IV; $n=4$, RR, 2.28; 95\% CI, 1.31-3.99; P=0.001). Taken together, these studies indicated that high expression levels of ALDH1 may be used to efficiently predict high WHO grade (III+IV) and poor outcome in patients with glioma. Thus, ALDH1 may be considered a novel pathological and prognostic hallmark for clinical use.

Several limitations of the present analysis should be acknowledged. Although ALDH1 expression in 5 of the 6 studies was detected by IHC, only 1 study was analyzed using TMA. IHC is a traditional method, the outcomes of which may be affected by different primary antibody clones and concentrations. It is impossible to perform sub-analysis with different antibodies. In addition, every factor that may affect the analysis should be fully considered.

As the major cause of bias in systematic meta-analyses, publication biases should be accordingly calculated. In the present study, publication bias was assessed by Begg's funnel plots test (27). This analysis indicated that there was no evident risk of publication bias in the meta-analysis. Other factors may also lead to bias, including language. The articles included in the present study were written only in English and Chinese, and therefore other potential studies were not included, which may also have led to bias.
In conclusion, based on the results obtained in the present study, this meta-analysis has shown that ALDH1 is a significant clinical biomarker in patients with glioma with poor prognosis and a high WHO grade (III+IV). These results indicated that ALDH1 has significance for pathological diagnosis and prognostic prediction of patients with glioma in clinical practice. In addition, given the limitations of the current analysis, well-designed prospective clinical studies are required to further evaluate its role in selecting therapeutic approach in glioma.

\section{Acknowledgments}

The present study was supported by Nature Foundation of China (grant nos. 81550028, and 81201766 to L.-L.Z. and J.W.), The Hubei Office of Education Foundation (grant nos. Q20151204 and B2016022 to L.-L.Z and J.W.), and The Yi Chang Office of Education Foundation (grant no. A16-301-14 to J.W.).

\section{References}

1. Cheng Y, Zhao J, Qiao W and Chen K: Recent advances in diagnosis and treatment of gliomas using chlorotoxin-based bioconjugates. Am J Nucl Med Mol Imaging 4: 385-405, 2014.

2. Krex D, Klink B, Hartmann C, von Deimling A, Pietsch T, Simon M, Sabel M, Steinbach JP, Heese O, Reifenberger G, et al: Long-term survival with glioblastoma multiforme. Brain 130: 2596-2606, 2007.

3. Nowak AK, Maujean JE, Jackson M and Knuckey N: A prospective study of surgical patterns of care for high grade glioma in the current era of multimodality therapy. J Clin Neurosci 18: 227-231, 2011.

4. Dalerba P, Cho RW and Clarke MF: Cancer stem cells: Models and concepts. Annu Rev Med 58: 267-284, 2007.

5. Jones RJ and Matsui W: Cancer stem cells: From bench to bed-side. Biol Blood Marrow Transplant 13 (Suppl 1): S47-S52, 2007.

6. Pallini R, Ricci-Vitiani L, Banna GL, Signore M, Lombardi D, Todaro M, Stassi G, Martini M, Maira G, Larocca LM and De Maria R: Cancer stem cell analysis and clinical outcome in patients with glioblastoma multiforme. Clin Cancer Res 14: 8205-8212, 2008.

7. Zeppernick F, Ahmadi R, Campos B, Dictus C, Helmke BM, Becker N, Lichter P, Unterberg A, Radiwimmer B and Herold-Mende CC: Stem cell marker CD133 affects clinical outcome in glioma patients. Clin Cancer Res 14: 123-129, 2008.

8. Huang Z, Cheng L, Guryanova OA, Wu Q and Bao S: Cancer stem cells in glioblastoma-molecular signaling and therapeutic targeting. Protein Cell 1: 638-655, 2010.

9. Laks DR, Visnyei K and Kornblum HI: Brain tumor stem cells as therapeutic targets in models of glioma. Yonsei Med J 51: 633-640, 2010

10. Marcato P, Dean CA, Giacomantonio CA and Lee PW: Aldehyde dehydrogenase: Its role as a cancer stem cell marker comes down to the specific isoform. Cell Cycle 10: 1378-1384, 2011.

11. Croker AK, Goodale D, Chu J, Postenka C, Hedley BD, Hess DA and Allan AL: High aldehyde dehydrogenase and expression of cancer stem cell markers selects for breast cancer cells with enhanced malignant and metastatic ability. J Cell Mol Med 13: 2236-2252, 2009.

12. Jiang F, Qiu Q, Khanna A, Todd NW, Deepak J, Xing L, Wang H, Liu Z, Su Y, Stass SA and Katz RL: Aldehyde dehydrogenase 1 is a tumor stem cell-associated marker in lung cancer. Mol Cancer Res 7: 330-338, 2009.

13. Rasper M, Schäfer A, Piontek G, Teufel J, Brockhoff G, Ringel F, Heindl S, Zimmer C and Schiegel J: Aldehyde dehydrogenase 1 positive glioblastoma cells show brain tumor stem cell capacity. Neuro Oncol 12: 1024-1033, 2010.

14. Friedman HS, Colvin OM, Kaufmann SH, Ludeman SM, Bullock N, Bigner DD and Griffith OW: Cyclophosphamide resistance in medulloblastoma. Cancer Res 52: 5373-5378, 1992. 
15. Quemener V, Moulinoux JP, Martin C, Darcel F, Guegan Y, Faivre J and Quash GA: Aldehyde dehydrogenase activity in xenografted human brain tumor in nude mice. Preliminary results in human glioma biopsies. J Neurooncol 9: 115-123, 1990.

16. Steels E, Paesmans M, Berghmans T, Branle F, Lemaitre F, Mascaux C, Meert AP, Vallot F, Lafitte JJ and Sculier JP: Role of p53 as a prognostic factor for survival in lung cancer: A systematic review of the literature with a meta-analysis. Eur Respir J 18: 705-719, 2001.

17. Tierney JF, Stewart LA, Ghersi D, Burdett S and Sydes MR: Practical methods for incorporating summary time-to-event data into meta-analysis. Trials 8: 16, 2007.

18. Parmar MK, Torri V and Stewart L: Extracting summary statistics to perform meta-analyses of the published literature for survival endpoints. Stat Med 17: 2815-2834, 1998.

19. Sterne JA and Egger M: Funnel plots for detecting bias in meta analysis: Guidelines on choice of axis. J Clin Epidemiol 54: 1046-1055, 2001

20. Adam SA, Schnell O, Pöschl J, Eigenbrod S, Kretzschmar HA Tonn JC and Schüller U: ALDH1A1 is a marker of astrocytic differentiation during brain development and correlates with better survival in glioblastoma patients. Brain Pathol 22: 788-797, 2012.

21. Campos B, Centner FS, Bermejo JL, Ali R, Dorsch K, Wan F, Felsberg J, Ahmadi R, Grabe N, Reifenberger G, et al: Aberrant expression of retinoic acid signaling molecules influences patient survival in astrocytic gliomas. Am J Pathol 178: 1953-1964, 2011.

22. Liu DY, Ren CP, Yuan XR, Zhang LH, Liu J, Liu Q, Yuan J, Yuan D and Jiang XJ: ALDH1 expression is correlated with pathologic grade and poor clinical outcome in patients with astrocytoma. J Clin Neurosci 19: 1700-1705, 2012.
23. Schäfer A, Teufel J, Ringel F, Bettstetter M, Hoepner I, Rasper M, Gempt J, Koeritzer J, Schmidt-Graf F, Meyer B, et al: Aldehyde dehydrogenase 1A1-a new mediator of resistance to temozolomide in glioblastoma. Neuro Oncol 14: 1452-1464, 2012.

24. Xu SL, Liu S, Cui W, Shi Y, Liu Q, Duan JJ, Yu SC, Zhang X, Cui YH, Kung HF and Bian XW: Aldehyde dehydrogenase 1A1 circumscribes high invasive glioma cells and predicts poor prognosis. Am J Cancer Res 5: 1471-1483, 2015.

25. Zeng LC, Ye F, Guo DS and Lei T: The expression of ALDH1 in glioma. Chin J Exp Surg 30: 1794-1795, 2013.

26. number> Moghbeli M, Moghbeli F, Forghanifard MM and Abbaszadegan MR: Cancer stem cell detection and isolation. Med Oncol 31: 69, 2014

27. Begg CB and Berlin JA: Publication bias: A problem in interpreting medical data. J Royal Statistical Soc 151: 419-463, 1988. 